\title{
Properties of predictive formulation of the Nambu-Jona-Lasinio model and ghost problem
}

\author{
V.E. Rochev \\ Institute for High Energy Physics, 142280, Protvino, Moscow region, Russia
}

\begin{abstract}
Recently proposed by Battistel et al. "predictive formulation of the NJL model" is discussed and its connection with the differential regularization is noted. The principal problem of this formulation is a non-physical singularity (Landau pole) in meson propagators. A modification of the formulation, which is free of the Landau pole and conserves main features of the approach, is proposed.
\end{abstract}

PACS numbers: 12.39.-x, 12.38.Bx, 11.30.Rd 
Effective models in the non-perturbative region are a considerable part of the modern stronginteraction theory. One of the most successful effective models of strong interaction of light hadrons is the Nambu-Jona-Lasinio (NJL) model with quark content. Since the NJl model in the leading approximation includes the quark loops and is based on non-renormalized four-fermion interaction, the essential aspect of this model is a regularization, which on the widely current opinion constitutes a part of the definition of the NJL model. Though predictions of the NJL model for commonly used regularizations (such as the cutoff in momentum space, the Pauli-Villars regularization or the proper-time regularization) are very similar (see, e.g., [2] for review), nevertheless such dependence on the regularization prescription cannot be satisfactory from the general theoretic point of view.

A very interesting attempt was made recently in the work of Battistel et al [1] for releasing the NJL model from the regularization dependence. In this work a method for the definition of one-loop Green functions of the NJL model was proposed (see also foregoing works [3]). The idea of the method is to avoid the explicit evaluation of divergent integrals with any specific regularization. The finite parts are separated of the divergent ones and are integrated without regularization. Then the NJL model becomes predictive in the sense that its consequences do not depend on the specific regularization of divergent integrals. An important result of work [1] is a proof of the fulfilment of all symmetry constraints on leading-order Green functions. A parameter choice leads to the reasonable values of the constituent quark mass and other model parameters.

In the present paper some features of the calculational scheme of work $[1]^{1}$ are analyzed, and a connection of the BDK approach with the differential regularization is pointed. The principal problem of the BDK approach is the singularity of meson propagators in the Euclidean momentum region. The presence of this singularity (Landau pole) prevents meson-loop calculations and, therefore, makes impossible any calculations of corrections to the leading approximation. A modification of the scheme, which is free of Landau pole and conserves the main features of the approach of [1], is proposed.

Following [1] we consider the simplest physically non-trivial variant of the NJL model with chiral symmetry $S U_{V}(2) \times S U_{A}(2)$. The model Lagrangian is

$$
\mathcal{L}=\bar{\psi}\left(i \hat{\partial}-m_{0}\right) \psi+\frac{g}{2}\left[(\bar{\psi} \psi)^{2}+\left(\bar{\psi} i \gamma_{5} \boldsymbol{\tau} \psi\right)^{2}\right] .
$$

Here $\psi$ is the quark field with $n_{c}$ colours, $m_{0}$ is the current quark mass, $g$ is the coupling constant of $m^{-2}$ dimension and $\boldsymbol{\tau}$ are Pauli matrices.

The leading approximation of the model is the mean-field approximation, which coincides in the case with the leading order of $1 / n_{c}$-expansion. All Green functions of the leading approximation are expressed in terms of quark one-loop integrals. A problem of calculations of these integrals is reduced to the definition of following five divergent integrals (see [1]):

$$
\begin{gathered}
\left\{I_{1} ; I_{1}^{\mu}\right\}=\int \frac{d^{4} k}{(2 \pi)^{4}} \frac{\left\{1 ; k^{\mu}\right\}}{\left(k+k_{1}\right)^{2}-M^{2}} \\
\left\{I_{2} ; I_{2}^{\mu} ; I_{2}^{\mu \nu}\right\}=\int \frac{d^{4} k}{(2 \pi)^{4}} \frac{\left\{1 ; k^{\mu} ; k^{\mu} k^{\nu}\right\}}{\left[\left(k+k_{1}\right)^{2}-M^{2}\right]\left[\left(k+k_{2}\right)^{2}-M^{2}\right]}
\end{gathered}
$$

Here $M$ is the dynamical (constituent) quark mass, which is a non-trivial solution of the gap equation for the NJL model.

A basic device for the definition of integrals (2) and (3) in the BDK approach is an algebraic identity for the propagator function (see equation (28) in work [1]). With the identity the divergent parts of integrals (2) and (3) have been expressed via three tensorial and two scalar external-momentum-independent integrals which were treated in the sense of some unspecified regularization. Then integrals (2) and (3) have been expressed in terms of these five integrals and two standard convergent integrals (see formulae of section III in work [1]).

\footnotetext{
${ }^{1}$ We shall name the calculational scheme of work [1] as the BDK approach, or the implicit regularization.
} 
In connection with these expressions we draw attention to some general regularization-independent property of integrals (2) and (3). Namely, integrals $\left\{I_{1} ; I_{1}^{\mu}\right\}$ and $\left\{I_{2} ; I_{2}^{\mu} ;\right\}$ are connected with following relations

$$
\frac{\partial I_{1}\left(k_{1} ; M^{2}\right)}{\partial M^{2}}=I_{2}\left(k_{1}, k_{1} ; M^{2}\right)
$$

and

$$
\frac{\partial I_{1}^{\mu}\left(k_{1} ; M^{2}\right)}{\partial M^{2}}=I_{2}^{\mu}\left(k_{1}, k_{1} ; M^{2}\right) .
$$

These relations are regularization independent and play a significant part in the approach. It is easy to verify the validity of these relations for traditional regularization schemes (four-dimensional cutoff, Pauli-Villars regularization, etc) by straightforward calculation, but their validity is not evident directly from above-mentioned formulae of work [1]. To prove these relations we should define derivatives of the external-momentum-independent integrals over $M^{2}$. For this purpose we note that derivatives of logarithmically-divergent integrals are convergent integrals and can be calculated without any regularization. The calculations of these convergent integrals gives us zero value for derivatives of tensorial logarithmically-divergent integrals. Then it is easy to verify that the derivatives of quadratically-divergent integrals are the corresponding logarithmically-divergent integrals. Taking into account these circumstances it is easy to prove equations (4) and (5) for BDK expressions of integrals (2) and (3). The significance of relations (4) and (5) are evident since they can be used for alternative definitions of quadratically divergent integrals $I_{1}$ and $I_{1}^{\mu}$ without additional regularization.

With obtained expressions for integrals (2) and (3) the authors of work [1] have analyzed the symmetry constraints on one-loop Green functions and have argued the necessity of consistency relations, which are concluded in zero value for tensorial external-momentum-independent integrals (see eq. (86) in work [1]). With these relations all symmetry properties of the theory (such as Furry theorem, Ward identities, etc) are fulfilled for all one-loop Green functions. This point is one of the important results of work [1]. From the point of view of above discussion the BDK consistency relations simply assert zero values of corresponding integration constants.

The choice of parameters of the NJL model with Lagrangian (1) in the leading approximation is principally defined by two divergent integrals, namely, logarithmically-divergent integral $I_{2}$ and quadratically-divergent integral $I_{1}$. Integral $I_{1}$ is a part of the gap equation

$$
M=m_{0}-8 i g n_{c} M \cdot I_{1},
$$

which defines dynamical (constituent) quark mass $M$. Integral $I_{2}$ determines the structure of meson propagators. Both these integrals can easily be defined with differential regularization without using the above-mentioned algebraic identity for the propagator function.

To define $I_{2}$ one can use well-known trick, which goes back to Gelfand and Shilov [4] (see also [5]). Namely, let introduce new external variables

$$
p=k_{1}-k_{2}, \quad P=k_{1}+k_{2} .
$$

Derivatives $\partial I_{2} / \partial p_{\mu} \partial I_{2} / \partial P_{\mu}$ are convergent integrals, and their calculation gives us

$$
\frac{\partial I_{2}}{\partial p_{\mu}}=\frac{i p_{\mu}}{16 \pi^{2}} \int_{0}^{1} d u \frac{u(1-u)}{M^{2}-u(1-u) p^{2}}, \quad \frac{\partial I_{2}}{\partial P_{\mu}}=0 .
$$

Basing on these results and using identity $p^{2} \frac{\partial f}{\partial p^{2}}=\frac{1}{2} p_{\mu} \frac{\partial f}{\partial p_{\mu}}$, we naturally go to the following definition:

$$
I_{2}\left(p^{2}\right)=-\frac{i}{16 \pi^{2}} \int_{0}^{1} d u \log \frac{M^{2}-u(1-u) p^{2}}{M_{0}^{2}}
$$


where $M_{0}$ is the integration constant. This expression coincide with the BDK ones if constant $M_{0}$ is related to the scalar external-momentum-independent integral $I_{l o g}$ (see eq. (40) in work [1]) as

$$
I_{l o g}=-\frac{i}{16 \pi^{2}} \log \frac{M^{2}}{M_{0}^{2}}=I_{2}(0) .
$$

An essential feature of the above calculation is the permutation of two limits - differentiation and regularization removing. Sure, such permutation is implied also in work [1] (in the calculations of finite parts). This permutation is an essence of Gelfand-Shilov differential regularization [4], which is based on the infinite differentiability of generalized functions.

To define $I_{1}$ we use regularization-independent relation (4), which gives (with taking into account eq. (10))

$$
I_{1}=\frac{i}{16 \pi^{2}}\left(M^{2} \log \frac{M_{0}^{2}}{M^{2}}+M^{2}-M_{1}^{2}\right),
$$

where $M_{1}^{2}$ is the integration constant of eq. (4). By taking into account the consistency relations such definitions also corresponds to BDK ones.

To calculate $M_{0}$ and $M_{1}$ and, therefore, to define $I_{0}$ and $I_{1}$ in full, we can use in the chiral limit $\left(m_{0}=0\right)$ two regularization-independent relations of NJL model (see, e.g. [2]), namely

$$
f_{\pi}^{2}=\frac{4 n_{c} M^{2}}{i} I_{2}(0)
$$

where $f_{\pi}$ is the pion-decay constant, and

$$
\chi=2 c^{3}=\frac{8 n_{c} M}{i} I_{1}
$$

where $\chi=<0|\bar{\psi} \psi| 0>$ and $c$ is the quark condensate. We have from equation (12)

$$
I_{2}\left(p^{2}\right)=\frac{i}{16 \pi^{2}}\left[\frac{4 \pi^{2} f_{\pi}^{2}}{n_{c} M^{2}}-\int_{0}^{1} d u \log \left(1-u(1-u) \frac{p^{2}}{M^{2}}\right)\right] .
$$

Then, using equations (11) and (13), we obtain for $M_{1}$

$$
M_{1}^{2}=M^{2}+\frac{4 \pi^{2} f_{\pi}^{2}}{n_{c}}-\frac{4 \pi^{2} c^{3}}{n_{c} M} .
$$

This equation (we shall refer it as BDK equation) plays a key role in the BDK approach. ${ }^{2}$

At pion constant $f_{\pi}$ and quark condensate $c$ being fixed equation (15) is the equation for dynamical quark mass $M$, where $M_{1}$ is a parameter. In terms of new variable $x=-\frac{\left(n_{c} / 2 \pi^{2}\right)^{1 / 3}}{c} M$ equation (15) can be written as

$$
x^{3}-3 a x+2=0,
$$

where $a=\frac{\left(n_{c} / 2 \pi^{2}\right)^{2 / 3}}{3 c^{2}}\left(M_{1}^{2}-\frac{4 \pi^{2} f_{\pi}^{2}}{n_{c}}\right)$. Depending on the value of parameter $a$, three cases are possible: (1) at $a<1$ equation (16) possesses one real negative root $x_{1}<0$;

(2) at $a=1$ equation (16) possesses one negative root $x_{1}=-2$ and one positive root $x_{2}=1$.

(3) at $a>1$ equation (16) possesses one negative root $x_{1}<0$ and two positive roots $0<x_{2}<1$ and $x_{3}>1$.

It is clear, that at $c<0$ the second case $(a=1)$ only can be physically accepted. The authors of work [1] take only this situation as a solely possible choice for model parameters. The value of the dynamical quark mass, which corresponds to positive root $x_{2}=1$ at $n_{c}=3$, is $M=-1.873 c$.

\footnotetext{
${ }^{2}$ An alternative way to introduce the implicit regularization can be based on an approach of work [6].
} 
At condensate value $c=-250 \mathrm{MeV}$ it gives $M=468 \mathrm{MeV}$. Coupling constant $g$ can be defined from the well-known relation of the NJL model

$$
g=-M / 2 c^{3},
$$

which is also regularization-independent (see, e.g., [2]). Noteworthy, the quark mass value and the coupling value depend on the quark condensate value only, and do not depend on the pion constant value. The last one defines the value of $M_{1}$, which is $M_{1}=879 \mathrm{MeV}$ at $f_{\pi}=93 \mathrm{MeV}$.

It is interesting to compare the parameter values of the BDK approach with the parameter values of traditional regularizations. The value of quark mass $(468 \mathrm{MeV})$ in the BDK approach is noticeably higher in comparison with the values in the four-dimensional momentum cutoff regularization $(236 \mathrm{MeV}$ ) and the Pauli-Villars regularization $(240 \mathrm{MeV}$ ) (at given value of quark condensate $-250 \mathrm{MeV}$ ). Apart from the quark mass dependence on the condensate value is quite different. In the BDK approach the quark mass is proportional to the condensate, whereas in 4-momentum cutoff and Pauli-Villars regularization the quark mass increases at decreasing the absolute value of condensate. For instance, at condensate value $c=-210 \mathrm{MeV}$ the quark mass is $M=393 \mathrm{MeV}$ in the BDK approach and $M=423 \mathrm{MeV}$ in the four-dimensional momentum cutoff. At the same time, from the point of view of the phenomenology the parameters' values in BDK approach are quite reasonable. Apart from it is necessary to recognize that expressions for the Green functions are much more simple in comparison with any traditional regularization.

In the framework of traditional regularizations the chiral-symmetry-breaking phase always coexists with the chiral-symmetric phase, which is energetically unfavored. The existence of the symmetric phase is not evident for the implicit regularization in the original formulation of work [1]. But the transition to the symmetric phase with $M=0$ (in chiral limit $m_{0}=0$ ) can be easily performed with the proposed differential formulation of the implicit regularization. Really, taking $M=0$ in equation (8), we obtain $\frac{\partial I_{2}}{\partial p_{\mu}}=-\frac{i p_{\mu}}{16 \pi^{2} p^{2}}$ and, therefore, $I_{2}\left(p^{2}\right)=-\frac{i}{8 \pi^{2}} \log \frac{p^{2}}{M_{0}^{2}}$ instead of (9). Then $I_{1}=-\frac{i M_{1}^{2}}{16 \pi^{2}}$ at $M \rightarrow 0$ and from equations (12) and (13) it follows that $f_{\pi} \rightarrow 0$ and $c \rightarrow 0$ in correspondence with the trivial physics of the symmetric phase.

An essential difference from other regularizations manifests the properties of Green functions in the Euclidean momentum region. In the BDK approach the meson propagators possess a nonphysical singularity - a pole at the negative momentum square (Landau pole, or Landau ghost). ${ }^{3}$ The meson propagators in the NJL model are scalar and pseudoscalar parts of the two-particle amplitude and can be written as

$$
D_{\sigma}=-\frac{i g}{m_{0} / M-4 i g n_{c}\left(4 M^{2}-p^{2}\right) I_{2}\left(p^{2}\right)}
$$

for the sigma-meson and

$$
D_{\pi}=-\frac{i g}{m_{0} / M+4 i g n_{c} p^{2} I_{2}\left(p^{2}\right)} .
$$

for the pion. In the chiral limit the equation for the Landau pole, as it follows from equation (14), is

$$
z \log \frac{z+1}{z-1}=2+\frac{1}{n_{c}}\left(\frac{2 \pi f_{\pi}}{M}\right)^{2},
$$

where $z=\sqrt{1-\frac{4 M^{2}}{p^{2}}}$. At above values of the model parameters $\left(n_{c}=3, f_{\pi}=93 \mathrm{MeV}, \mathrm{M}=\right.$ $468 \mathrm{MeV})$ this equation has solution $p_{L}^{2}=-4.29 \mathrm{M}^{2}=-(969 \mathrm{MeV})^{2}$, i.e., Landau mass value

\footnotetext{
${ }^{3}$ The existence of such pole was discovered firstly in quantum electrodynamics [7] and presents a characteristic feature of theories without an asymptotic freedom in deep-Euclidean region. The existence of the Landau ghost in a system of fermions coupled to a chiral field has been observed in work [8]
} 
$M_{L}^{2}=-p_{L}^{2}$ approximately twice larger in comparison with the quark mass: $M_{L}=2.07 M$. Beyond the chiral limit (at $m_{0} \neq 0$ ) this value changes very small (about 1\%) due to the smallness of current quark mass $m_{0}$.

The existence of the Landau pole is a serious problem of the BDK approach. In particular, any calculations with meson loops become problematic. Though such calculations exceed the framework of the one-loop approximation, which is a subject of work [1], the impracticability of these calculations means a principal impossibility of calculations of corrections to leading approximation and cannot be acceptable.

Further, the Landau pole in quantum electrodynamics due to the smallness of fine structure constant $\alpha$ is located in the very distant asymptotic Euclidean region $\left(\left(M_{L}^{2}\right)^{Q E D} \simeq-m_{e}^{2} \exp \left\{\frac{3 \pi}{\alpha}\right\}\right.$, where $m_{e}$ is the electron mass and $\left.\alpha \simeq 1 / 137\right)$, and its presence can be in principle ignored. Really, at the much smaller energies the quantum electrodynamics becomes a part of an asymptotically free grand unification theory with self-consistent asymptotic behavior. In the NJL model with the implicit regularization the Landau pole is located near the physical region of the model, and similar reasoning is impossible in principle. From the other hand, the traditional regularizations, such as the four-dimensional cutoff or the Pauli-Villars regularization, are free on this problem the meson propagators in these regularizations do not have the Landau poles. Therefore, implicit regularization, possessing the certain appeal and simplicity, contains serious defect as the nearby Landau pole.

To improve the situation a compromise approach is necessary, which conserves main features of implicit regularization and at the same time solves the problem of the Landau pole. Such compromise can be achieved with the Feynman regularization for logarithmically divergent integral $I_{2}$ :

$$
I_{2}\left(p^{2}\right)=\int \frac{d^{4} q}{(2 \pi)^{4}}\left\{\frac{1}{\left(M^{2}-(p+q)^{2}\right)\left(M^{2}-q^{2}\right)}-\frac{1}{\left(M_{r}^{2}-(p+q)^{2}\right)\left(M_{r}^{2}-q^{2}\right)}\right\},
$$

where $M_{r}$ is a regulator mass $\left(M_{r}^{2}>M^{2}\right)$, and the definition of quadratically divergent integral $I_{1}$ as before is made by relation (4).

In Euclidean region $p^{2}<0$ integral (21) can be represented as

$$
I_{2}=\frac{i}{16 \pi^{2}} F\left(-\frac{p^{2}}{M^{2}}\right)
$$

where

$$
F(x)=\int_{0}^{1} d u \log \frac{M_{r}^{2} / M^{2}+u(1-u) x}{1+u(1-u) x} .
$$

Prove the absence of zeroes of this function at $x>0$. Evaluating elementary integrals in equation (22) and introducing variables $v=\frac{1}{2}\left(\sqrt{1+\frac{4}{x}}-1\right)$ and $v_{r}=\frac{1}{2}\left(\sqrt{1+\frac{4 M_{r}^{2}}{x M^{2}}}-1\right)$ we obtain the following expression:

$$
F=2\left[\log \frac{1+v_{r}}{1+v}+v_{r} \log \left(1+\frac{1}{v_{r}}\right)-v \log \left(1+\frac{1}{v}\right)\right] .
$$

If $M_{r}^{2}>M^{2}$, then $v_{r}>v$, and from elementary inequalities $\log \frac{1+v_{r}}{1+v}>0$ and $v_{r} \log \left(1+\frac{1}{v_{r}}\right)>$ $v \log \left(1+\frac{1}{v}\right)$ it follows that $F>0$ in the Euclidean region, i.e. the meson propagators do not possess the Landau pole with this definition. For other aspects this modified regularization is similar to implicit regularization. For the modified regularization

$$
I_{2}(0)=-\frac{i}{16 \pi^{2}} \log \frac{M^{2}}{M_{r}^{2}},
$$

and, consequently, $I_{1}$ is defined by same formula (11) with substitution

$$
M_{0} \rightarrow M_{r},
$$


i.e. integration constant $M_{0}$ everywhere is substituted by regulator mass $M_{r}$. Such re-definition of $I_{1}$ still implies an existence of additional parameter $M_{1}^{2}$, which is the integration constant of equation (4).

Equation (15), which determines quark mass $M$, has exactly the same form for this modified regularization, and, consequently, all parameter values are the same. Remark, condition $M_{r}^{2}>M^{2}$ is the automatical consequence of formula (12). Therefore, the proposed modification conserves main features of the implicit regularization and simultaneously solves the problem of Landau pole. ${ }^{4}$

A general receipt for definitions of divergent integrals (2) and (3) can be formulated as follows: integrals (3) are defined by formulae

$$
\left\{I_{2} ; I_{2}^{\mu} ; I_{2}^{\mu \nu}\right\}=\left\{I_{2} ; I_{2}^{\mu} ; I_{2}^{\mu \nu}\right\}^{B D K}\left(M^{2}\right)-\left\{I_{2} ; I_{2}^{\mu} ; I_{2}^{\mu \nu}\right\}^{B D K}\left(M_{r}^{2}\right) .
$$

Upper index $B D K$ means that each integral with mass $M$ or $M_{r}$ is defined by formulae of work $[1] .^{5}$

Then integrals (2) are defined by equations (4) and (5). A consistency relation is the coincidence of integration constants, and, therefore,

$$
I_{1}^{\mu}=-k_{1}^{\mu} I_{1}
$$

as in the BDK approach with consistency conditions.

It should be noted, that due to linearity of consistency constraints considered in work [1], the analysis of symmetry conservation, which was made in this work, can be carried to the proposed modification.

A principal distinction between the implicit regularization and the proposed ghostless modification consists in the treating of integral $I_{2}$. Parameter $M_{0}$ in the implicit regularization is the integration constant whereas parameter $M_{r}$ in the Feynman regularization (21) is introduced "by hands" and seems to be an outside object. The question arises, if really this object is some "secret parameter", which ensures the self-consistency of the NJL model on the quantum level? We cannot answer to this question now with the full confidence, but the structure of the implicit regularization gives us an allusion to the possible realization of this idea. The term with regulator mass $M_{r}$ in the definition of integral $I_{2}$ by formula (21) can be interpreted as the rise of some repulsive interaction (i.e. interaction with a negative coupling) at the one-loop level. The coupling constant of the NJL model is connected with the quark mass and the condensate by formula (17). If the quark condensate is negative the negative coupling corresponds to the negative quark mass. In this connection it is not out of place to recall about the negative solution of BDK equation (15) ("quark ghost"). In the physical case this solution is $-2 M$, where $M$ is the dynamical quark mass. The existence of this solution can be an indication of such repulsive interaction, which becomes apparent on the one-loop quantum level. At given above parameter values we have $\left|M_{r}\right| \simeq 1.3 M$. This value, of course, is far from the expected value. When the absolute value of the quark condensate decreases ratio $\left|M_{r}\right| / M$ arises, and at $c=-210 \mathrm{MeV}$ it equals to $\simeq 1.5$. At $c=-150 \mathrm{MeV}$ it reaches expected value 2 , but the last value of the condensate is phenomenologically unacceptable since it leads to the very large value of the current quark mass $m_{0}=21 \mathrm{MeV}$. Therefore, the question of the possible "inter-termination of ghosts" in the framework of the NJL model remains to be open.

\footnotetext{
${ }^{4}$ Another way to remove the Landau ghost in the NJL model is the method of work [9], based on the KallenLehmann representation. This method has been applied in work [10] to the chiral $\sigma$-model. However, the proposed receipt seems to be preferable in the framework of the implicit regularization since this regularization has deal with a set of integrals while the method of works [9, 10] should be applied to the proper two-point functions.

${ }^{5}$ With such definitions integrals $I_{2}$ and $I_{2}^{\mu}$ are convergent without additional regularization. Since for selfconsistency of the receipt it is necessary to verify the coincidence of usual calculation of these integrals with the calculation by formula (25), based on the BDK definition of divergent integrals. Simple calculation demonstrates the reality of such coincidence.
} 
In conclusion, discuss briefly the definition of model parameters beyond the chiral limit. At $m_{0} \neq 0$ from eq.(19) it follows the pion spectrum equation

$$
m_{0}=\frac{2 i n_{c} M^{2}}{c^{3}} \cdot m_{\pi}^{2} \cdot I_{2}\left(m_{\pi}^{2}\right)
$$

Practically this equation is used for definition of current quark mass $m_{0}$.

Taking approximation $I_{2}\left(m_{\pi}^{2}\right) \simeq I_{2}(0)$, we obtain the well-known current-algebraic Gell-MannOakes-Renner formula

$$
m_{\pi}^{2} f_{\pi}^{2} \simeq-m_{0}<\bar{\psi} \psi>
$$

This relation, of course, is regularization-independent (and what is more, model-independent). At $m_{\pi}=135 \mathrm{MeV}, c=-250 \mathrm{MeV}$ we obtain from eq.(28) $m_{0}=5.2 \mathrm{MeV}$. The exploiting of regularization-dependent exact value $I_{0}\left(m_{\pi}^{2}\right)$ changes this result very slightly (approximately of 1 percent) due to the smallness of pion mass, and such specification, of course, lays out the framework of the model, it being right as for the implicit regularization as the modified variant.

The sigma-meson mass in the chiral limit is $2 M$. Beyond the chiral limit the equation on the sigma-meson mass follows from equation (18):

$$
m_{0}=\frac{2 i n_{c} M^{2}}{c^{3}} \cdot\left(m_{\sigma}^{2}-4 M^{2}\right) \cdot I_{2}\left(m_{\sigma}^{2}\right) .
$$

With rather crude approximation $I_{2}\left(m_{\sigma}^{2}\right) \simeq I_{2}(0)$ and with taking into account equation (28) we obtain the well-known regularization-independent formula (see, e.g., [2])

$$
m_{\sigma}^{2} \simeq 4 M^{2}+m_{\pi}^{2}
$$

The much more exact (but regularization-dependent) approximation is $I_{2}\left(m_{\sigma}^{2}\right) \simeq I_{2}\left(4 M^{2}\right)$. In this case, we obtain

$$
m_{\sigma}^{2} \simeq 4 M^{2}+0.21 m_{\pi}^{2}
$$

for the implicit regularization, and

$$
m_{\sigma}^{2} \simeq 4 M^{2}+0.26 m_{\pi}^{2}
$$

for the modified variant.

As we see from the above formulae, in the physical region of energies about $1 \mathrm{GeV}$ the quantitative difference of these regularizations is not very significant.

\section{Acknowledgments}

Author is grateful to R Jafarov and V A Petrov for useful discussions.

\section{References}

[1] Battistel O A, Dallabona G and Krein G 2008 Phys.Rev. D 77065025 (hep-th/0803.0537)

[2] Klevansky S P 1992 Rev.Mod.Phys. 64649

[3] Battistel O A and Nemes M C 1999 Phys.Rev. D 59 055010;

Farias R L S, Dallabona G, Krein G and Battistel O A 2006 Phys.Rev. C 77065201

[4] Gelfand I M and Shilov G E 1964 Generalized Functions (Academic, San Diego) Vol. 1

[5] Collins J C 1984 Renormalization (Cambridge Univ. Press) 
[6] Farhi E, Graham N, Jaffe R L and Weigel H 2002 Nucl.Phys. B 630241

[7] Landau L D and Pomeranchuk I Ya 1955 Dokl.Akad.Nauk Ser.Fiz. 102489

[8] Ripka G and Kahana S 1987 Phys.Rev. D 361233

[9] Redmond P J 1958 Phys.Rev. 1958 1404;

Bogoliubov N N, Logunov A A and Shirkov D V 1959 ZhETF 37805

[10] Hartmann J, Beck F and Bentz W 1994 Phys.Rev C 503088 